Topological Methods in Nonlinear Analysis

Journal of the Juliusz Schauder Center

Volume 3, 1994, 163-178

\title{
THE POINCARÉ-BENDIXSON THEOREMS FOR TWO-DIMENSIONAL SEMIFLOWS
}

\author{
KRZYSZTOF CIESIELSKI ${ }^{1}$
}

Dedicated to Jean Leray

\section{Introduction}

The Poincaré-Bendixson theory plays an important role in the study of qualitative behaviour of autonomous differential equations and dynamical systems on $\mathbb{R}^{2}$. It describes very precisely the structure of limit sets in such systems. Many theorems are named after Poincaré and Bendixson; the most classical one says that when a semitrajectory is bounded and its limit cycle does not contain stationary points, then the limit cycle is a periodic trajectory.

After the famous Bendixson work of 1901, many generalizations and improvements of the theory were obtained. The theorems were generalized to 2-manifolds (either directly or in a slightly changed version); it was proved that under some regularity assumptions only certain particular situations may occur ([18]). Other theorems of Poincaré-Bendixson type were proved (especially [10]), sometimes even for non-2-dimensional phase space (cf. [21]). It was shown that the theorems may be generalized to flows (dynamical systems), without differentiability assumptions (see [11]).

1991 Mathematics Subject Classification. 54H20, 58F21, 34C35.

${ }^{1}$ Supported by Polish Scientific Grant 658/2/91. 
The theory of semiflows (semidynamical systems) has been developed since the publication of the book [1]. Generally, semiflows have a much more complicated structure than flows since the movement is defined only in the positive direction.

In this paper we prove that the description of limit sets given by the PoincaréBendixson theorems is also true for semiflows. However, this is not a simple generalization of similar theorems for flows, as all the known proofs of the PoincaréBendixson theorems depend on the uniqueness of negative semi-solutions and the continuity of movement in both directions. Thus, according to the results presented in this paper, we see that the characterization of limit sets given by the Poincaré-Bendixson theorems is not only purely topological and does not depend on differentiability assumptions, but even depends only on the movement defined for positive values of the time variable $t$. Moreover, in semiflows there are defined negative semisolutions and negative limit sets given by these solutions. Of course the points of the phase space do not need have negative unicity; different solutions through one point may behave in different ways and give different limit sets. Generally, negative continuations admit complicated situations which are impossible in the case of flows. Nevertheless, we show that also in this negative case a suitable "Poincaré-Bendixson type" characterization of limit sets is true.

The proofs use many topological properties and theorems from the theory of semiflows. One of the key results is the existence of sections in semiflows ([5]), which enables us to give a precise description of suitable neighbourhoods of an element of the phase space. This description leads to some properties of limit sets, which yield the required characterization. Some methods used in this paper are not new. In particular, in some places we apply earlier techniques due to Hajek ([11]), Serra and Tarallo ([20]), Ciesielski ([6]), Ciesielski and Omiljanowski ([7]). Note that if we assume that a semillow is a flow, then the proof presented here is another proof of the Poincaré-Bendixson theorems, in some places even simpler than the known ones, but in some places more complicated as semiflows have a more complicated structure.

In Section 2 we set up notation and terminology. Section 3 is devoted to the study of sections on 2-manifolds. Section 4 presents the results on intersections of sections and limit sets, which lead to main results contained in Section 5 . In this section we prove the Poincaré-Bendixson theorems for semiflows and give some corollaries describing 2-dimensional semiflows.

For the basic properties of flows and semiflows the reader is referred to $[1]$, [2], [11] and [17]. Some results and examples particularly interesting from the point of view of this paper can also be found in [3], [4], [8], [15], [19] and [22]. The theorems in the case of differential equations are fully described for instance 
in [12], [14] and [23]. Further information on topological results used here can be found in [9], [13], [16] and [24].

\section{Preliminaries}

Throughout this paper we assume that $M$ is a given 2-manifold (without boundary); we also admit noncompact manifolds.

By an arc (a Jordan curve) we mean a homeomorphic image of the compact interval $[-1,1]$ (a unit circle). A homeomorphism $\varphi:[-1,1] \rightarrow T$ will be called a parametrization of an arc $T$. By $\overline{a b}$ we denote an arc with end-points $a, b$. A 2-manifold $M$ is called dichotomic if any Jordan curve cuts $M$ into two open connected domains. For a given set $X$ we denote its interior by $\operatorname{Int} X$ and its closure by $\mathrm{Cl} X$.

A semiflow (semidynamical system) on $M$ (which is called a phase space) is a triplet $\left(M, \mathbb{R}_{+}, \pi\right)$ where $\pi: \mathbb{R}_{+} \times M \rightarrow M$ is a continuous function such that $\pi(0, x)=x$ and $\pi(t, \pi(u, x))=\pi(t+u, x)$ for any $t, u, x$. Replacing $\mathbb{R}_{+}$by $\mathbb{R}$ we get a definition of a flow (dynamical system).

Assume that $\left(M, \mathbb{R}_{+}, \pi\right)$ is a given semiflow.

We denote $\bigcup\{\pi(t, x): t \geq 0\}$ by $\pi^{+}(x)$ and we set $\pi^{+}(A)=\bigcup\left\{\pi^{+}(x): x \in\right.$ $A\}$. We put $F(t, x)=\{y \in M: \pi(t, y)=x\}$ and $F(\Delta, A)=\bigcup\{F(u, y): u \in$ $\Delta, y \in A\}$ for $A \subset M, \Delta \subset \mathbb{R}_{+}$. We write $F(\Delta, x)$ instead of $F(\Delta,\{x\})$.

A solution through $x$ is usually defined as a function $\sigma: \Delta \rightarrow M$ where $\Delta$ is an interval containing 0 such that $\sigma(0)=x$ and $\pi(t, \sigma(u))=\sigma(t+u)$ for any $t, u$ with $u \in \Delta, t \geq 0, t+u \in \Delta$. If a solution $\sigma$ is maximal (relative to the property of being a solution, with respect to inclusion), then its image is called a trajectory through $x$. Note that in such case $[0, \infty)$ is contained in the domain of solution. According to the main results of [15] and [1, Theorem 11.8] we may assume without loss of generality that any solution can be prolongated so as to have $(-\infty, 0]$ contained in its domain. This is because we can transform the system by a suitable isomorphism which does not change trajectories (and therefore their topological properties), but only changes the speed of movement along trajectories. In this paper by a solution (through $x$ ) we mean a solution with a domain equal to $\mathbb{R}$, and consequently trajectories are the images of such solutions. By a positive (negative) semisolution through $x$ we mean the suitable solution defined on $[0, \infty)((-\infty, 0])$; their images are called positive (negative) semitrajectories. Note that for any $x$ there is precisely one positive semisolution through $x$, however there may exist an infinite number of negative semisolutions through $x$.

A set $A$ is positively invariant if $\pi^{+}(A)=A$. A set $A$ is negatively invariant if $F([0, \infty), A)=A$. We call the set $A$ weakly negatively invariant if for any $x \in A$ there is a negative semisolution $\sigma$ through $x$ with $\sigma(-\infty, 0] \subset A$. 
A set $A$ is positively (weakly) minimal if it is nonempty, closed and positively (weakly negatively) invariant and does not contain any proper subset having all these properties.

We put $L^{+}(x)=\left\{y \in M: \pi\left(t_{n}, x\right) \rightarrow y\right.$ for some $\left.t_{n} \rightarrow \infty\right\}$ and $L_{\sigma}^{-}(x)=$ $\left\{y \in M: \sigma\left(t_{n}\right) \rightarrow y\right.$ for some $\left.t_{n} \rightarrow-\infty\right\}$, where $\sigma$ is a negative semisolution through $x$, and call them a positive and negative limit set, respectively. Generally we call all these sets limit sets. Note that for a given point $x$, different negative semisolutions can give different negative limit sets. It is known ([1]) that limit sets in semiflows on manifolds are positively and weakly negatively invariant.

A point $x$ is said to be

- stationary if $\pi(t, x)=x$ for every $t \geq 0$,

- periodic if there exists a $t>0$ such that $\pi(t, x)=x$ and $x$ is not stationary,

- eventually periodic if $\pi(t, x)$ is periodic for some $t>0$,

- eventually stationaty if $\pi(t, x)$ is stationary for some $t>0$.

A solution (semi-solution, trajectory, semi-trajectory) given by a stationary, periodic or eventually periodic point is called stationary, periodic or eventually periodic, respectively.

A closed set $S$ containing $x$ is called a $\lambda$-section (for short: a section) through $x$ if there is a closed set $T$ such that:

(a) $F(\lambda, T)=S$,

(b) $F([0,2 \lambda], T)$ is a neighbourhood of $x$,

(c) $F(\mu, T) \cap F(\nu, T)=\emptyset$ for $0 \leq \mu<\nu \leq 2 \lambda$.

We will call the set $T$ a bar.

This definition generalizes the classical definition of a section in dynamical systems. The basic properties of sections in semiflows are established in [5]. In particular, it is proved there that for any nonstationary point $x$ there exists a section through $x$. Note that from the definition and [7] it follows immediately that a section in a semiflow on a 2-manifold cannot reduce to a one-point set. By a transversal we mean a section which is simultaneously an arc. A $\lambda$-transversal is a transversal which is simultaneously a $\lambda$-section. When $S$ is a transversal with end-points $a, b$, we denote by $S^{0}$ the set $S \backslash\{a, b\}$.

Under our assumptions on solutions it is easy to check that $F(t, F(u, x))=$ $F(t+u, x)$ and $F([u, t], x)=\pi([0, t-u], F(t, x)$ for any $t, u, x$.

Throughout this paper we assume that $\left(M, \mathbb{R}_{+}, \pi\right)$ is a given semiflow on a 2-manifold $M$. Recall that we assume that any solution is defined on $\mathbb{R}$, as this involves no loss of generality. 


\section{The characterization of sections}

THEOREM 3.1. Assume that $S$ is a compact connected $\lambda$-section. Then $S$ is an arc or a Jordan curve.

Proof. We will show that $S$ has an open base each of whose elements has a boundary with at most two points. Then from classical theorems characterizing arcs and Jordan curves ([13, Ch. 47 and 51]) it follows that $S$ must be a Jordan curve or an arc, as $S$ is a continuum containing more than one point.

Take an $x \in S$ and a neighbourhood $U$ of $x$. Take a $\gamma>0$ such that $\gamma<\lambda$. According to $[1$, Theorem 4.4] we can choose $\gamma$ with $F([0, \gamma], x) \cup \pi([0, \gamma], x) \subset U$. The set $F(\gamma, x)$ is a point or an $\operatorname{arc}([7$, Theorem 3.4]). Take a $y \in F(\gamma, x)$. Using the Schönflies Theorem we may assume that $J=\pi([0,2 \gamma], y)$ is a diameter of an open disc $D$ contained in $U$. The mapping $\pi(\cdot, y)$ is one-to-one on $[0,2 \gamma]$. Note that $S \cap J=\{x\}$, as $S$ is a $\lambda$-section. Denote the components of $D \backslash J$ by $D_{1}$ and $D_{2}$. We have:

if $z \in D_{i}$ and $\pi([0, \delta], z) \subset D$, then $\pi([0, \delta], z) \subset D_{i} \cup J$ for $i=1,2$. Moreover, if $\pi([0, \delta], z) \cap J \neq \emptyset$, then $\pi([0, \delta], z) \cap J=\pi([\alpha, \delta], z)$ for some $\alpha$.

From the definition of section, suitable continuity properties of semiflows ([1, $4.4],[3,1.7,2.9])$ and again $[7,3.4]$ we deduce that there are a $u \in(0, \gamma)$ and a set $G$ homeomorphic to an open disc such that:

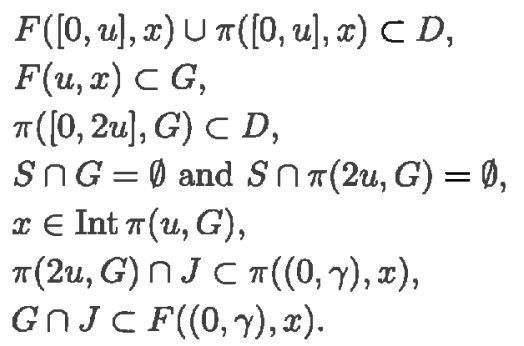

Assume that $D_{i} \cap S \cap \operatorname{Int} \pi(u, G) \neq \emptyset$ for $i=1,2$ (if one of these sets is empty, the proof is analogous but simpler). Take $x_{1}, x_{2}$ belonging to these sets, respectively and a $y_{i} \in G$ with $\pi\left(u, y_{i}\right)=x_{i}$ (see Figure 1). Note that $\pi\left([0,2 u], y_{i}\right) \cap J \subset \pi((0, \gamma), x)$. There is an arc $\overline{y_{1} y_{2}} \subset G$ such that $\overline{y_{1} y_{2}} \cap J$ is a one-point set. The set $\pi(2 u, G)$ is arcwise connected, so there are $z_{1}, z_{2} \in J$ and arcs with $\overline{\pi\left(2 u, y_{i}\right) z_{i}} \subset \pi(2 u, G), \overline{\pi\left(2 u, y_{i}\right) z_{i}} \cap J=\left\{z_{i}\right\}$ for $i=1,2$ (it may happen that $z_{i}=\pi\left(2 u, y_{i}\right)$ and in this case $\overline{\pi\left(2 u, y_{i}\right) z_{i}}$ reduces to one point). By $\overline{z_{1} z_{2}}$ we denote the suitable segment contained in $J$. Define:

$$
\begin{aligned}
& C_{1}=\overline{\pi\left(2 u, y_{1}\right) z_{1}} \cup \overline{z_{1} z_{2}} \cup \overline{\pi\left(2 u, y_{2}\right) z_{2}} \subset \pi(2 u, G), \\
& C_{2}=\overline{y_{1} y_{2}} \cup \pi\left([0,2 s],\left\{y_{1}, y_{2}\right\}\right), \\
& C=C_{1} \cup C_{2} .
\end{aligned}
$$

The set $C$ is a compact subset of $D, x \notin C$ and, according to the properties of $G, C \cap S=\left\{x_{1}, x_{2}\right\}$, as $S$ is a section. Take the component $X$ of $M \backslash C$ with 


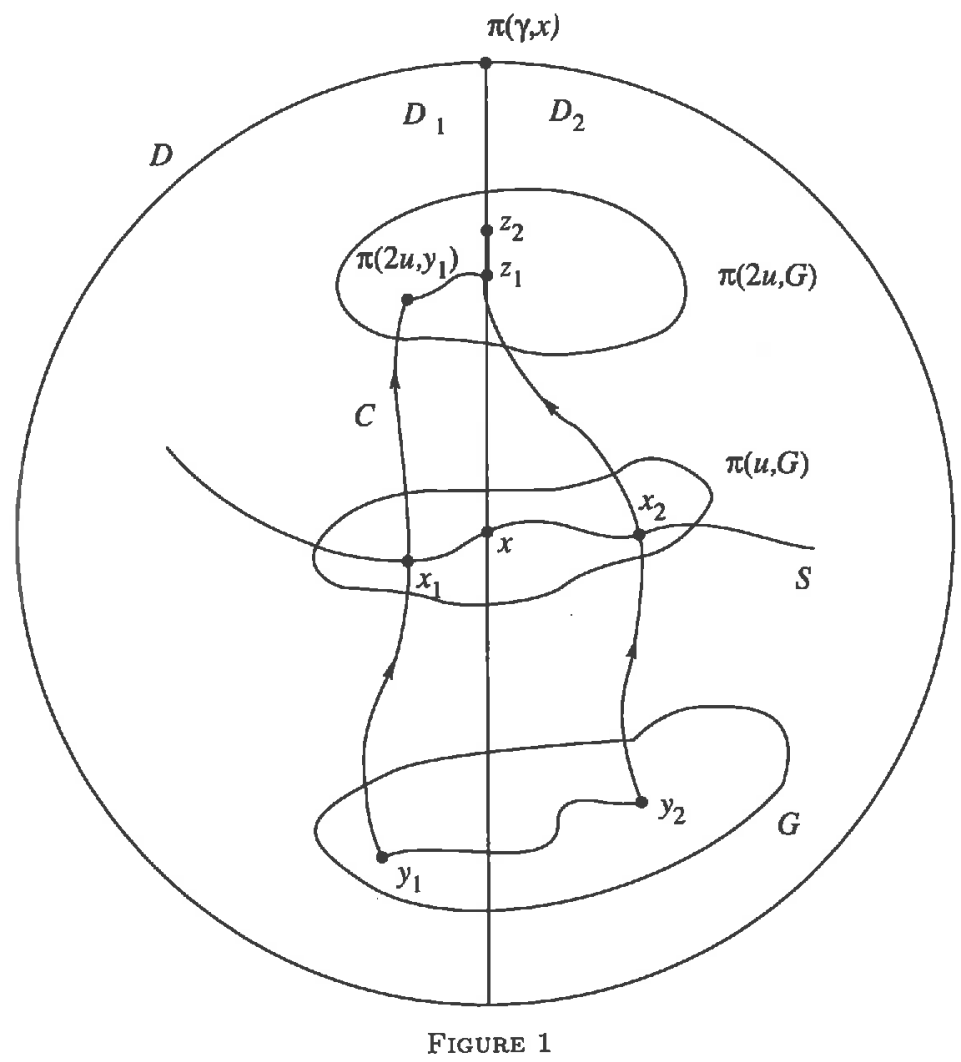

$x \in X$. From the construction we see that $X \cap S$ is the required neighbourhood of $x$ in $S$, as the boundary of $X \cap S$ in $S$ is equal to $\left\{x_{1}, x_{2}\right\}$.

From the above theorem and [5, Theorem 2.5] we get easily

THEOREM 3.2. For every nonstationary point $x \in M$ there exists a transversal through $x$.

In order to present the theorem characterizing precisely small neighbourhoods given by sections we show the following

LEMMA 3.3. If $S$ is a transversal, then $F(\lambda, S)$ is connected.

Proof. The set $F(\lambda, S)$ is compact ([15, Theorem 3.8]). Suppose it is not connected, i.e. there are nonempty closed disjoint sets $A$ and $B$ with $A \cup B=$ $F(\lambda, S)$. Therefore $\pi(\lambda, A \cup B)=S$, the sets $\pi(\lambda, A)$ and $\pi(\lambda, B)$ are compact and closed in $S$, so there is an $x \in \pi(\lambda, A) \cap \pi(\lambda, B)$, because $S$ is connected. This gives that $F(\lambda, x) \cap A \neq \emptyset$ and $F(\lambda, x) \cap B \neq \emptyset$, which is impossible, as $F(\lambda, x)$ is a point or an arc $([7,3.4])$, hence a connected set. 
THEOREM 3.4. For any nonstationary point $x$ there is a $\lambda$-transversal $S$ through $x$ with a bar $T(F(\lambda, T)=S)$ such that

(3.4.1) $T$ is an arc,

(3.4.2) $F(\mu, T)$ is a transversal (through $\pi(\lambda-\mu, x)$ for $\mu \in[0, \lambda]$, through any point of $F(\mu-\lambda, x)$ for $\mu \in[\lambda, 2 \lambda])$,

(3.4.3) $F([0,2 \lambda], T)$ is homeomorphic to a square and the boundary of $F([0,2 \lambda]$, $T)$ is equal to $T \cup F(2 \lambda, T) \cup \pi([0,2 \lambda],\{a, b\})$, where by $a, b$ we denote the end-points of the arc $F(2 \lambda, T)$,

(3.4.4) $F([0,2 \lambda], T) \backslash S$ has two components: $F([0, \lambda), T)^{\circ}=\pi((0, \lambda], S)$ and $F((\lambda, 2 \lambda], T)=F((0, \lambda], S)$.

Proof. Take any transversal $S$ through $x$ (Theorem 3.2). By $[5,1.10]$ we can find a $\lambda$ and a $T$ such that $F(\lambda, T)=S$ and $F(\mu, T)$ is a section required in (3.4.2) for $\mu \in[0,2 \lambda]$; $T$ is a bar for the $\lambda$-section $S$. Let $p, q$ be the end-points of $S$. We may choose $\lambda$ with $\pi([0, \lambda], p) \cap \pi([0, \lambda], q)=\emptyset$.

By Theorem 3.1 and Lemma 3.3 we know that $F(\mu, T)$ is an arc or a Jordan curve. We show that the latter is impossible. Take $p_{\nu}, q_{\nu} \in F(\nu, S)$ with $\pi\left(\nu, p_{\nu}\right)=p, \pi\left(\nu, q_{\nu}\right)=q$. Suppose that $F(\nu, S)$ is a Jordan curve; thus it is equal to the union of two different arcs with end-points $p_{\nu}$ and $q_{\nu}$. The image of each of these arcs under the continuous transformation $\pi(\nu, \cdot)$ is equal to $\overline{p q}=S$, so we can find an arc $J$ (which is a transversal) contained in $\overline{p q} \backslash\{p, q\}$ with $F(\nu, J)$ not connected. This contradicts Lemma 3.3. On the other hand, if $\pi(\nu, S)$ is a Jordan curve then it is the union of two different arcs with end-points $\pi(\nu, p)$ and $\pi(\nu, q)$. Therefore, by Lemma 3.3, the inverse image of each such arc by $\pi(\nu, \cdot)$ is equal to $\overline{p q}$, which is impossible. The contradiction proves (3.4.2) and (3.4.1), as $\pi(\lambda, S)=T$ and $S$ is a $\lambda$-transversal.

Denote $F(2 \lambda, T)$ by $K$. According to [5] we have $F([0,2 \lambda], T)=\pi([0,2 \lambda], K)$; it is clear that this set is connected.

Now consider the mapping $h=\left.\pi\right|_{[0,2 \lambda] \times K}$ and take any $z \in F([0,2 \lambda], T)$. Note that $h^{-1}(\{z\})=\{\nu\} \times F(\nu, z)$ for some (precisely one) $\nu$, so, as above, it is a compact connected set. It is easily seen that the set $F([0,2 \lambda], T) \backslash\{z\}$ is connected. From [24, Cor. IX.2.41] used for the mapping $h$ we conclude that (3.4.3) is fulfilled.

From (3.4.3), using $[5,1.7]$ and the $\Theta$-Curve Theorem ([13], [16]) we get (3.4.4) and finish the proof.

\section{Intersections of transversals and limit sets}

Throughout this section we assume that the 2-manifold $M$ is dichotomic. We assume that a nonstationary point $x \in M$ and a $\lambda$-transversal $S$ through $x$ are given, where $\lambda$ is taken as in Theorem 3.4. 
LEMMA 4.1. Assume that $\sigma$ is a solution through $x$ and $t$ is a real number with $\sigma(t) \in S$. Then $[\sigma(t-\lambda, t) \cup \sigma(t, t+\lambda)] \cap S=\emptyset$. In other words, the points of $S$ are isolated on $\sigma(\mathbb{R})$ with respect to the time variable $t$.

This is an immediate consequence of Theorem 3.4.

LEMMA 4.2. Assume that $\sigma$ is a solution through $x$. Take a $t_{1}<t_{2}$ with $\sigma\left(t_{i}\right) \in S$, for $i=1,2$ and $\sigma\left(t_{1}, t_{2}\right) \cap S=\emptyset$. Let $\varphi:[-1,1] \rightarrow S$ be $a$ homeomorphism with $\varphi\left(s_{i}\right)=\sigma\left(t_{i}\right)$ for some $s_{1}, s_{2} \in[-1,1]$. We may assume that $s_{1} \leq s_{2}$. Define a Jordan curve $\Gamma$ to be $\sigma\left[t_{1}, t_{2}\right] \cup \varphi\left[s_{1}, s_{2}\right]$. Then $M \backslash \Gamma$ has two open components $\Omega^{+}$and $\Omega^{-}$such that $\mathrm{Cl} \Omega^{+}$is positively invariant and $\Omega_{-}$is negatively invariant (Figure 2). Moreover, if $s_{1}<s_{2}$, then $\varphi\left(s_{2}, 1\right] \subset \Omega^{+}, \varphi\left[-1, s_{1}\right) \subset \Omega^{-}, \sigma\left(t_{2}, \infty\right) \cap \varphi\left(s_{1}, s_{2}\right)=\emptyset$ and $\sigma\left(-\infty, t_{1}\right) \subset \Omega^{-}$. If $s_{1}=s_{2}$ then the set $S \cap \pi^{+}\left(\sigma\left(t_{1}\right)\right)$ has precisely one element and we may fix the parametrization $\varphi$ with $\varphi\left[-1, s_{1}\right) \subset \Omega^{-}, \varphi\left(s_{2}, 1\right] \subset \Omega^{+}$.

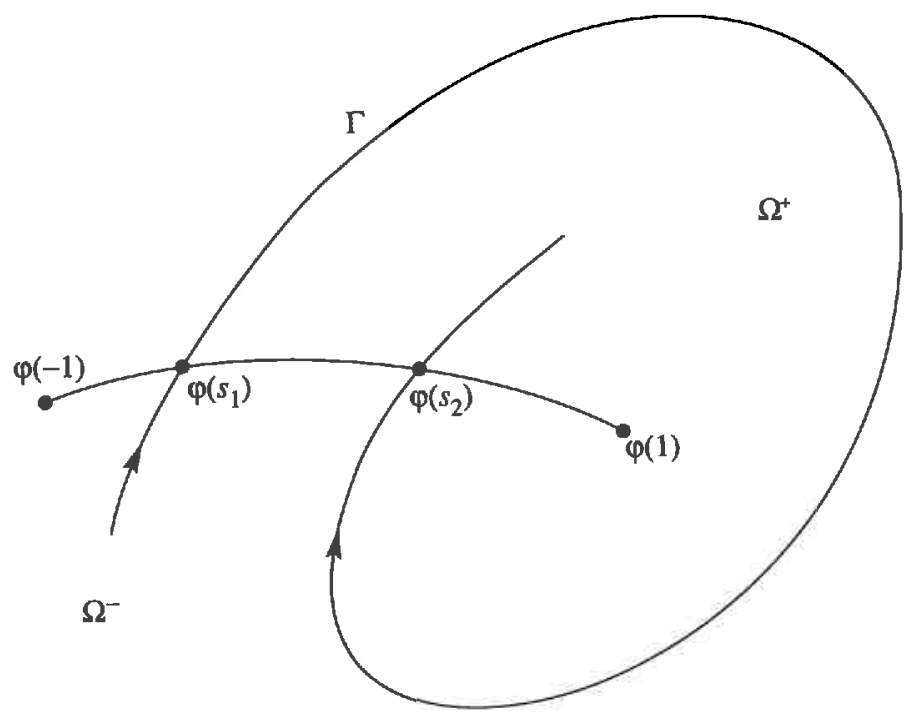

Figure 2

Proof. First assume that $s_{1}<s_{2}$. Denote by $T$ a bar of the $\lambda$-transversal $S$. Using the Schönflies Theorem we may assume that $S=\varphi[-1,1]$ is a segment. For any $s \in\left(s_{1}, s_{2}\right)$ there is an open disc $U_{s}$ centred at $\varphi(s)$ such that $U_{s} \cap \Gamma=\emptyset$ and $U_{s} \subset \operatorname{Int} F([0,2 \lambda], T)$. Denote by $U_{s}^{+}$the component of $U_{s} \backslash S$ which is contained in $F([0, \lambda), T)$ and by $U_{s}^{-}$the other component (cf. Theorem 3.4). Put $U^{+}=\bigcup\left\{U_{s}^{+}: s \in\left(s_{1}, s_{2}\right)\right\}$ and $U^{-}=\bigcup\left\{U_{s}^{-}: s \in\left(s_{1}, s_{2}\right)\right\}$. It is easy to notice that for $s \neq w$ the sets $U_{s}^{+}$and $U_{w}^{+}$are contained in the same component of $M \backslash \Gamma$ and an analogous property holds for $U_{s}^{-}$and $U_{w}^{-}$. Denote the components of $M \backslash \Gamma$ by $\Omega^{+}$and $\Omega^{-}$, let $U^{+} \subset \Omega^{+}$and $U^{-} \subset \Omega^{-}$. 
We show that $\mathrm{Cl} \Omega^{+}$is positively invariant. According to the definition of $\Gamma$, Theorem 3.4 and the construction of $U^{+}$we see that $\pi\left((0, \delta), \varphi\left(s_{2}\right)\right) \subset \Omega^{+}$for $\delta \in(0, \lambda)$. Now suppose on the contrary that there exists a $y \in \mathrm{Cl} \Omega^{+}$and a $u_{0}$ with $\pi\left(u_{0}, y\right) \in \Omega^{-}$. Thus we may find a $u<u_{0}$ such that $\pi(u, y) \in \Gamma$ and $\pi\left(\left(u, u_{0}\right), y\right) \subset \Omega^{-}$. We have:

$$
\begin{array}{ll}
\pi(u, y) \notin \varphi\left(s_{1}, s_{2}\right) & \left(\text { as } U^{+} \subset \Omega^{+}\right), \\
\pi(u, y) \notin \sigma\left[t_{1}, t_{2}\right) & \left(\text { as } \sigma\left[t_{1}, t_{2}\right) \subset \Gamma\right), \\
\pi(u, y) \neq \sigma\left(t_{2}\right)=\varphi\left(s_{2}\right) & \left(\text { as } \pi\left((0, \delta), \varphi\left(s_{2}\right)\right) \subset \Omega^{+}\right),
\end{array}
$$

so $\pi(u, y) \notin \Gamma$, which is a contradiction.

Now $\Omega^{-}=M \backslash C l \Omega^{+}$is negatively invariant, which finishes the proof of the first part of Lemma 4.2 .

By the definition of $\Gamma$ we have either $\varphi\left(s_{2}, 1\right] \subset \Omega^{+}$or $\varphi\left(s_{2}, 1\right] \subset \Omega^{-}$. Suppose that $\varphi\left(s_{2}, 1\right] \subset \Omega^{-}$. Consider $\left\{w_{n}\right\} \subset\left(s_{2}, 1\right]$, with $w_{n} \rightarrow s_{2}$. From Lemma 4.1 it follows that $\pi\left(\delta / 2, \varphi\left(w_{n}\right)\right) \in \Omega^{+}$for large $n$ (with $\delta$ as above); thus $\pi\left(\alpha, \varphi\left(w_{k}\right)\right) \in \Gamma$ for some $k$ and $\alpha \in(0, \delta / 2)$. However, $\pi\left(\alpha, \varphi\left(w_{k}\right)\right) \notin S$, so $\pi\left(\alpha, \varphi\left(w_{k}\right)\right) \in \sigma\left(t_{1}, t_{2}\right)$ and $\pi\left(\beta, \varphi\left(w_{k}\right)\right)=\varphi\left(s_{2}\right)$ for some $\beta \in(\alpha, \delta / 2)$, which contradicts Lemma 4.1 as $\beta<\lambda$. We have shown that $\varphi\left(s_{2}, 1\right] \subset \Omega^{+}$.

Now we prove that $\varphi\left[-1, s_{1}\right) \subset \Omega^{-}$. Suppose, on the contrary, that $\varphi\left[-1, s_{1}\right)$ $\subset \Omega^{+}$. Take a $\gamma<t_{2}-t_{1}$ such that (as in Theorem 3.4) $\pi\left(\gamma, \varphi\left[s_{1}, s_{2}\right]\right.$ ) is a transversal. Then $\Gamma \cup \pi\left([0, \gamma], \varphi\left(s_{2}\right)\right) \cup \pi\left(\gamma, \varphi\left[s_{1}, s_{2}\right]\right)$ forms a $\Theta$-curve and $\pi\left([0, \gamma], \varphi\left[s_{1}, s_{2}\right]\right)$ is a neighbourhood of $\varphi\left(s_{1}\right)$ in $\mathrm{Cl}^{+}$. This gives that for $\left\{w_{n}\right\} \subset\left[-1, s_{1}\right)$ with $w_{n} \rightarrow s_{1}$, we have $\varphi\left(w_{n}\right) \in \pi\left((0, \gamma], \varphi\left[s_{1}, s_{2}\right]\right)$ for large $n$, which is impossible as $\gamma<\lambda$.

Now we show that $\sigma\left(t_{2}, \infty\right) \cap \varphi\left(s_{1}, s_{2}\right)=\emptyset$. If not, $\sigma(t) \in \varphi\left(s_{1}, s_{2}\right)$ for some $t>t_{2}$ and from the construction of $U^{-}$we get $\sigma(t-\beta, t) \subset U_{\sigma(t)}^{-} \subset \Omega^{-}$for small $\beta$. However, for $t-\beta>t_{2}$ we have $\sigma(t-\beta) \in \mathrm{Cl} \Omega^{+}$, as $\mathrm{Cl} \Omega^{+}$is positively invariant.

In order to prove that $\sigma\left(-\infty, t_{1}\right) \subset \Omega^{-}$it is enough to show that $\sigma\left(t_{1}-\right.$ $\left.\mu, t_{1}\right) \subset \Omega^{-}$for some $\mu$, as $\Omega^{-}$is negatively invariant. But for a small $\mu$ we have $\sigma\left(t_{1}-\mu, t_{1}\right) \cap \Gamma=\emptyset$ and the proof follows by the same method as the proof that $\varphi\left[-1, s_{1}\right) \subset \Omega^{-}$.

We have shown the theorem for $s_{1}<s_{2}$. When $s_{1}=s_{2}$ the proof is similar but much simpler ( $\sigma\left[t_{1}, t_{2}\right]$ is equal to a periodic trajectory).

LemMa 4.3. Assume that $\varphi:[-1,1] \rightarrow S$ is a parametrization of $S$ and $\sigma$ is a solution with $\sigma\left(u_{i}\right)=\varphi\left(\alpha_{i}\right), i=1,2,3$, and $u_{1}<u_{2}, \alpha_{1}<\alpha_{2}$. Then:

- if $\sigma$ is injective, then $u_{2}<u_{3} \Leftrightarrow \alpha_{2}<\alpha_{3}$,

- if $\sigma$ is a solution through an eventually periodic point, then $\alpha_{2}<\alpha_{3} \Rightarrow$ $u_{2}<u_{3}$ and $u_{2}<u_{3} \Rightarrow \alpha_{2} \leq \alpha_{3}$. 
Proof. Let $u_{2}<u_{3}$. By Lemma 4.1 there are $t_{1}<t_{2}<\ldots<t_{n}$ such that $t_{1}=u_{1}, t_{k}=u_{2}$ for some integer $k \in(1, n), u_{3}=t_{n}$ and $\sigma\left(t_{i}\right)$ are the only points of $S \cap \sigma\left[u_{1}, u_{3}\right]$. Let $\sigma\left(t_{i}\right)=\varphi\left(s_{i}\right)$, so $\alpha_{2}=s_{k}$ and $\alpha_{3}=s_{n}$. Using Lemma 4.2 we obtain $s_{2} \leq s_{3}$ (as $\pi^{+}\left(\sigma\left(t_{2}\right)\right) \subset \mathrm{Cl} \Omega^{+}$) and we continue in this fashion to obtain $s_{k} \leq s_{n}$. If $\sigma$ is injective we get $s_{k}<s_{n}$. Similarly we show that if $\alpha_{2}<\alpha_{3}$ then $u_{2}<u_{3}$.

LEMMA 4.4. Let $\sigma$ be an injective semisolution with domain $[0, \infty)$ or $(-\infty, 0]$ and let $\varphi:[-1,1] \rightarrow S$ be a parametrization of $S$. Set $A=\{t: \sigma(t) \in S\}$ and define $f: A \rightarrow[-1,1]$ by $f(t)=\varphi^{-1}(\sigma(t))$. Then $f$ is a monotonic function.

The proof follows directly from Lemma 4.3.

THEOREM 4.5. If $\Lambda$ is a limit set, then $\Lambda \cap S^{0}$ has no more than one element.

Proof. Consider $\Lambda=L_{\sigma}^{-}(z)$ for some $z$ and $\sigma$; for $\Lambda=L^{+}(z)$ the proof is analogous.

Step 1. We show that for any $y \in \Lambda \cap S^{0}$ there exists $\left\{t_{n}\right\}$ with $t_{n} \rightarrow-\infty$, $\sigma\left(t_{n}\right) \rightarrow y$ and $\left\{\sigma\left(t_{n}\right)\right\} \subset S$. Let $T$ be a bar of the $\lambda$-transversal $S$; we have $F(\lambda, T)=S$. Let $\sigma\left(u_{n}\right) \rightarrow y$ for $u_{n} \rightarrow-\infty$. Then, by Theorem 3.4, $\sigma\left(u_{n}\right) \in$ $F([0,2 \lambda], T)$ for large $n$ and we can find a $\left\{t_{n}\right\}$ with $\left|u_{n}-t_{n}\right| \leq \lambda$ and $\sigma\left(t_{n}\right) \in S$. We can assume, taking a subsequence if necessary, that $\sigma\left(t_{n}\right) \rightarrow p \in S$. It is easy to observe that $\left|u_{n}-t_{n}\right| \rightarrow 0$, so $p=y$ and $t_{n} \rightarrow-\infty$.

Step 2. Suppose that $y, \widetilde{y} \in S^{0} \cap \Lambda, y=\varphi(\alpha), \widetilde{y}=\varphi(\widetilde{\alpha})$ and $y \neq \widetilde{y}$. Let $\alpha<\widetilde{\alpha}$. According to Step 1, there are sequences $\left\{t_{n}\right\},\left\{\widetilde{t}_{n}\right\}$ with $t_{n} \rightarrow-\infty, \widetilde{t}_{n} \rightarrow-\infty$ and $\sigma\left(t_{n}\right) \rightarrow y$ and $\sigma\left(\tilde{t}_{n}\right) \rightarrow \widetilde{y}$. Let $\sigma\left(t_{n}\right)=\varphi\left(\alpha_{n}\right)$ and $\sigma\left(\tilde{t}_{n}\right)=\varphi\left(\tilde{\alpha}_{n}\right)$. We can find disjoint neighbourhoods $U, \widetilde{U}$ of $y, \widetilde{y}$ such that if $\varphi(\beta) \in S \cap U$ and $\varphi(\widetilde{\beta}) \in S \cap \widetilde{U}$ then $\beta<\widetilde{\beta}$. Thus there is an integer $m_{0}$ such that $\varphi\left(\alpha_{k}\right)<\varphi\left(\widetilde{\alpha_{n}}\right)$ for any $k, n>m_{0}$. According to Lemma 4.4, $t_{k}<\widetilde{t}_{n}$ for any $k, n>m_{0}$. Hence $\left\{t_{n}\right\}$ and $\left\{\widetilde{t}_{n}\right\}$ cannot converge to $-\infty$ simultaneously. This contradiction shows that $S^{0} \cap \Lambda$ has at most one element.

THEOREM 4.6. Let $\Lambda$ be a limit set. Then for any nonstationary $y \in \Lambda$ and $t>0$ the set $\Lambda \cap F(t, y)$ has precisely one element.

Proof. According to $[1,5.6,5.15], \Lambda$ is weakly negatively invariant, so $\Lambda \cap F(t, y) \neq \emptyset$. Suppose that $z_{1}, z_{2} \in \Lambda \cap F(t, y), z_{1} \neq z_{2}$. There is an $\alpha \in(0, t]$ with $\pi\left([0, \alpha), z_{1}\right) \cap \pi\left([0, \alpha), z_{2}\right)=\emptyset$ and $\pi\left(\alpha, z_{1}\right)=\pi\left(\alpha, z_{2}\right)$. Now take an $\varepsilon$-transversal $R$ through $\pi\left(\alpha, z_{1}\right)$ satisfying the conditions from Theorem 3.4. Then $F(\beta, R)$ is a transversal and $\pi\left(\alpha-\beta, z_{i}\right) \in \operatorname{Int} F([0, \varepsilon], R)$ for a sufficiently small $\beta$ and $i=1,2$. Then $\pi\left(\alpha-\beta, z_{i}\right) \in F(\beta, R)^{0} \cap \Lambda$ as $\Lambda$ is positively invariant $([1,5.4,5.15])$, which contradicts Theorem 4.5. 
THEOREM 4.7. If a limit set $\Lambda$ does not contain any stationary point, then the semidynamical system $\left(\Lambda, \mathbb{R}_{+},\left.\pi\right|_{\mathbb{R}_{+} \times \Lambda}\right)$ induced on $\Lambda$ is a semidynamical system with negative unicity. Thus, if we define $\pi(t, x)$ for any $t<0$ and $x \in \Lambda$ in an obvious way, we get a dynamical system $\left(\Lambda, \mathbb{R},\left.\pi\right|_{\mathbb{R} \times \Lambda}\right)$.

The proof follows immediately from the invariance and weak invariance of $\Lambda$ $([1,5.4,5.6,5.15])$, Theorem 4.6 and $[3,2.12]$.

Using Theorem 4.6 we also obtain

CoRollary 4.8. No limit set $\Lambda$ contains a trajectory homeomorphic to the "figure-of-six" (see [17]), i.e. a nonperiodic but eventually periodic trajectory.

EXAMPLE 4.9. It is possible to construct a semiflow with a limit set $\Lambda$ containing a stationary point, without negative unicity in $\Lambda$ (see Figure $3 ; \Lambda$ is a circle, $\Lambda=L^{+}(x), y$ and $z$ are stationary, other points in $\Lambda$ are eventually stationary).

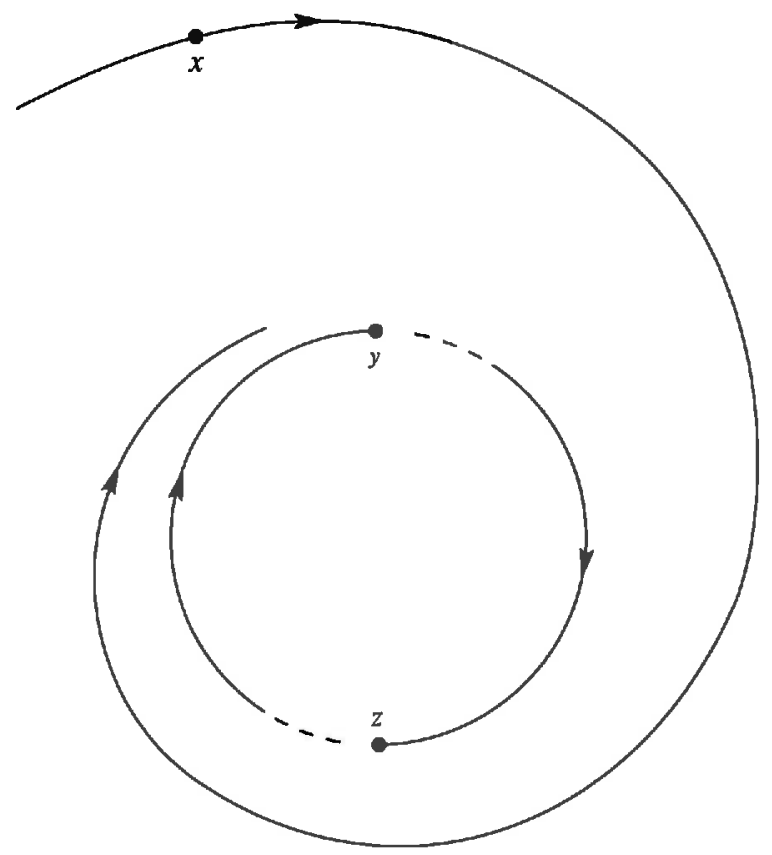

Figure 3

Remark 4.10. Theorem 4.6 is not true in higher dimensions. Put $Y=$ $\left\{\left(x, f_{1}(x), 0\right): x \in \mathbb{R}\right\} \cup\left\{\left(c, f_{2}(x), 0\right): x \in \mathbb{R}\right\} \subset \mathbb{R}^{3}$, where $f_{1}(x)=\max \{-x, 0\}$, $f_{2}(x)=\min \{x, 0\}$, and $\left.\pi\left(t, f_{i}(x), 0\right)\right)=\left(x+t, f_{i}(x+t), 0\right), i=1,2$. Now it is easy to construct a set $J$ homeomorphic to $\mathbb{R}, J=\sigma(\mathbb{R}) \subset \mathbb{R}^{2} \times(0, \infty)$ and extend a semiflow $\pi$ to $\mathbb{R}_{+} \times(Y \cup J)$ so as to get a semiflow on $Y \cup J \subset \mathbb{R}^{3}$ with $\sigma$ being a solution and $L^{+}(p)=Y$ for any $p \in J$. 


\section{The characterization of limit sets}

Throughout this section we assume that the 2-manifold $M$ is dichotomic.

LEMMA 5.1. Let $\Lambda$ be a limit set. If there is a periodic point $x \in \Lambda$ then $\Lambda=\pi^{+}(x)$.

Proof. Consider any $p \in \pi^{+}(x)$ and let $\sigma$ be a periodic solution through p. Take a $\lambda_{p}$-transversal through $p$ with a bar $B_{p}$, where $\lambda_{p}$ is taken as in Theorem 3.4. The set $\sigma(\mathbb{R})=\pi^{+}(x)$ is compact, so we can find $p_{1}, \ldots, p_{n} \in \sigma(\mathbb{R})$ with $\pi^{+}(x) \subset \bigcup\left\{\operatorname{Int} F\left(\left[0,2 \lambda_{p_{i}}\right], B_{p_{i}}\right): i=1, \ldots, n\right\}$. Moreover, according to Theorems 3.4, 4.5 and $4.7, \Lambda \cap \operatorname{Int} F\left(\left[0,2 \lambda_{p_{i}}\right], B_{p_{i}}\right) \subset \sigma\left(-\lambda_{p_{i}}, \lambda_{p_{i}}\right)$ for any $i$. Thus

$$
\Lambda \cap \bigcup\left\{\operatorname{Int} F\left(\left[0,2 \lambda_{p_{i}}\right], B_{p_{i}}\right): i=1, \ldots, n\right\}=\pi^{+}(x)
$$

which means that $\pi^{+}(x)$ is a component of $\Lambda$. Using $[1,5.5,5.11,5.15]$ we conclude that $\Lambda=\pi^{+}(x)$, as $\pi^{+}(x)$ is compact.

Now we present theorems which are generalizations of the classical PoincaréBendixson theorems.

THEOREM 5.2. If a limit set $\Lambda$ is connected and does not contain stationary points, then $\Lambda$ is a single trajectory.

\section{Proof.}

Step 1. According to Theorem 4.7, the function $\pi$ defines a dynamical system on $\Lambda$. Let us consider two topologies on $\Lambda$. The topology $\tau_{E}$ is induced from the topology on $M$. The topology $\tau_{\pi}$ is given by taking for a neighbourhood base at $y \in \Lambda$ the family $\left\{\sigma(-\delta, \delta): \delta \in\left(0, \lambda_{y}\right)\right\}$ where $\sigma$ is the unique solution through $y$ with $\sigma(\mathbb{R}) \subset \Lambda$ and $\lambda_{y}$ is taken from Theorem 3.4. We prove that $\tau_{E}=\tau_{\pi}$.

By Theorems 3.4, 4.5, 4.6 and 4.7, for any sufficiently small $\delta$ there is a euclidean neighbourhood $U$ of $y$ with $\sigma(-\delta, \delta)=U \cap \Lambda$. Thus $\tau_{\pi} \subset \tau_{E}$. On the other hand, transform a small neighbourhood $U$ of $y$ using the Schönflies Theorem in such way that $\sigma(-\alpha, \alpha)$ is a segment for some $\alpha$. Take a family of open balls $B_{r}$ with $r \in\left(0, r_{0}\right)$ for a given sufficiently small $r_{0}$. Using again Theorems 3.4, 4.5 and 4.7 we see that $B_{r} \cap \Lambda=\sigma\left(-\alpha_{1}, \alpha_{2}\right)$ for some $\alpha_{1}, \alpha_{2}>0$. This gives $\tau_{E} \subset \tau_{\pi}$.

Step 2. By the assumption and Step $1, \Lambda$ is connected in the topology $\tau_{\pi}$. For $y \in \Lambda$ denote by $\sigma_{y}$ the unique solution through $y$ with $\sigma_{y}(\mathbb{R}) \subset \Lambda$. By Theorem 4.7, $\left\{\sigma_{y}(\mathbb{R}): y \in \Lambda\right\}$ is a family of pairwise disjoint sets. Clearly, for any $y \in \Lambda$ the set $\sigma_{y}(\mathbb{R})$ is open in $\tau_{\pi}$; thus $\Lambda=\bigcup\left\{\sigma_{y}(\mathbb{R}): y \in \Lambda\right\}$ must be a single trajectory, as $\Lambda$ is connected in $\tau_{E}=\tau_{\pi}$.

THEOREM 5.3. Assume that the phase space $M$ is equal to $\mathbb{R}^{2}$. If a semitrajectory (positive or negative) is bounded, then a limit set $\Lambda$ associated with this 
trajectory is a single periodic trajectory provided that it does not contain any stationary point.

Proof. The limit set $\Lambda$ is bounded, hence compact and (by $[1,5.5,5.15]$ ) connected. From the previous theorem we conclude that $\Lambda$ is a single trajectory given by a solution $\sigma$. We only need to show that $\sigma$ is periodic. On account of Step 1 of the proof of Theorem 5.2 we know that $\Lambda$ is compact in $\tau_{\pi}$ and $\{\sigma(-n, n): n \in \mathbb{N}\}$ is a cover of $\Lambda$. Then $\sigma(\mathbb{R})=\Lambda \subset \sigma(-k, k)$ for some $k$, which means that $\sigma$ is not injective and contains a periodic trajectory. Applying Corollary 4.8 we finish the proof.

THEOREM 5.4. If a negative semisolution $\sigma$ through $y$ is contained in any limit set and $\sigma$ is not periodic, then $L_{\sigma}^{-}(y)$ may only contain stationary points.

Proof. Assume that $z \in L_{\sigma}^{-}(y)$ is not stationary. According to Theorems 3.4 and 4.5 there are $\mathrm{a} \lambda>0$ and a $\lambda$-section $S$ through $z$ with a bar $T$ such that $F([0,2 \lambda], T) \cap \Lambda \supset \rho[-\lambda, \lambda]$ and $\Lambda \cap F(\lambda, T)=\rho(0)$ for some solution $\rho$ through $z$. However, by Theorem $4.7 \pi$ induces on $\Lambda$ a semiflow with negative inicity, so $F([0,2 \lambda], T) \cap \Lambda=\rho[-\lambda, \lambda]$. Thus there is a sequence $\left\{t_{n}\right\}$ with $\sigma\left(t_{n}\right) \rightarrow-\infty$ and $\sigma\left(t_{n}\right) \rightarrow z$, so $\sigma\left(t_{n}\right) \in F([0,2 \lambda], T)$ for large $n$. Hence we have $\sigma\left(u_{n}\right) \in S^{0}$ for some $\left\{u_{n}\right\}\left(\left|u_{n}-t_{n}\right|<\lambda, u_{n} \rightarrow-\infty\right)$. It is clear that $\left|u_{n}-t_{n}\right| \rightarrow 0$ and $\sigma\left(u_{n}\right) \rightarrow z$. Theorem 4.5 now yields $\sigma\left(u_{n}\right)=z$, as $\sigma(-\infty, 0]$ is a subset of a limit set. This gives that $\sigma$ is periodic.

In the same manner (using positive invariance of limit sets and Corollary 4.8) we prove

THEOREM 5.5. If $y$ belongs to a limit set and $y$ is not periodic, then $L^{+}(y)$ may only contain stationary points.

The following corollary is a simple consequence of the three previous theorems and Lemma 5.1.

Corollary 5.6. Assume that the phase space $M$ is $\mathbb{R}^{2}$ and let a semitrajectory (positive or negative) be bounded. Then either the limit set $\Lambda$ associated with this trajectory is a periodic trajectory, or any semitrajectory contained in $\Lambda$ may contain in its limit set only stationary points.

EXAMPLE 5.7. The example presented below shows that (unlike the case of dynamical systems) Theorem 5.4 does not remain true if we replace the condition " $\sigma(y) \subset \Lambda$ " by " $y \in \Lambda$ " (see Figure 4). We have $x_{2} \in L_{\sigma}^{-}\left(x_{1}\right), x_{3} \in L_{\sigma}^{-}\left(x_{2}\right)$ and so on, $x_{1}, x_{2}, x_{3}, \ldots$ are periodic, all points contained in the annulus between $\pi^{+}\left(x_{i}\right)$ and $\pi^{+}\left(x_{i+1}\right)$ are eventually periodic. 


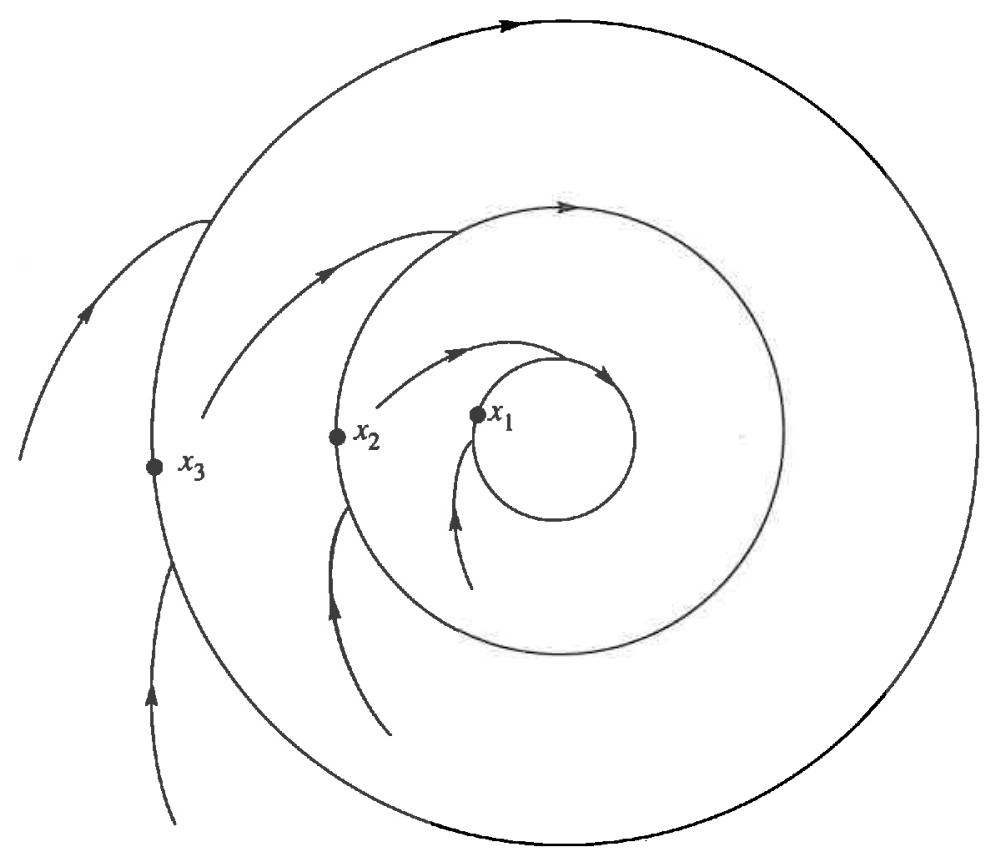

Figure 4

THEOREM 5.8. If $x \in L^{+}(x)$ (or $x \in L_{\sigma}^{-}(x)$ ) then $x$ is either stationary or periodic.

PROOF. For $x \in L^{+}(x)$ we obtain the required condition using Theorem 5.5.

Now let $x \in L_{\sigma}^{-}(x)$ and suppose that $\sigma$ is neither stationary nor periodic. Using Theorem 3.4, take a transversal $S$ through $x$; as in the proof of Theorem 5.4 there is a $\left\{u_{n}\right\} \subset(-\infty, 0)$ with $u_{n} \rightarrow-\infty, \sigma\left(u_{n}\right) \in S$ and $\sigma\left(u_{n}\right) \rightarrow x$. Put $t_{2}=0$ (so $\sigma\left(t_{2}\right)=x$ ) and $t_{1}<0$ with $\sigma\left(t_{2}\right) \in S$ and $\sigma\left(t_{1}, t_{2}\right) \cap S=\emptyset$. Let $\varphi$ be a parametrization of $S$ with $\varphi\left(s_{i}\right)=\sigma\left(t_{i}\right), i=1,2$. Now, by Lemma 4.2, $\sigma\left(u_{n}\right) \in$ $\Omega^{-}$(where $\Omega^{-}$is defined as in Lemma 4.2) for large $n$ and $\sigma\left(u_{n}\right) \in \varphi\left[-1, s_{1}\right.$ ), so $\sigma\left(u_{n}\right)$ cannot converge to $x=\varphi\left(s_{2}\right)$.

THEOREM 5.9. If a compact set $A$ is either positively minimal or weakly minimal, then it is either a stationary point or a periodic trajectory.

Proof. By $[1,12.3,12.9]$ we get $L^{+}(x)=x$ for any $x$ in $A$. From Theorem 5.8 we conclude that $x$ is either stationary or periodic and $A$ is either a stationary point or a periodic trajectory.

The existence of stationary points in flows and semiflows is usually an important problem. We end with the following

THEOREM 5.10. Assume that $M$ is a plane or a 2-dimensional sphere. If in the semiflow there exists a nonempty limit set, then at least one point of the phase space is stationary. 
First we show a useful

LEMMA 5.11. If a positively invariant set $B \subset M$ is homeomorphic to a closed disc, then $B$ contains a stationary point.

Proof. For any $n$ the mapping $\pi\left(\frac{1}{n}, \cdot\right): B \rightarrow B$ contains a fixed point $x_{n}$, according to the Brouwer Fixed Point Theorem. We can assume that $x_{n}$ converges to $x \in B$, as $B$ is compact. We show that $x$ is stationary. Indeed, take any $t>0$. Then for any $n$ there is an integer $k_{n}$ with $\frac{k_{n}}{n} \leq t<\frac{k_{n}+1}{n}$. Hence $\frac{k_{n}}{n} \rightarrow t, \pi\left(\frac{1}{n}, x_{n}\right)=\pi\left(\frac{k_{n}}{n}, x_{n}\right) \rightarrow \pi(t, x), \pi\left(\frac{1}{n}, x_{n}\right) \rightarrow x$, which implies $\pi(t, x)=x$.

Proof of THEOREM 5.10. Suppose on the contrary that no point of $\mathbb{R}^{2}$ is stationary. Assume that $\Lambda$ is a nonempty limit set of $x$ and take a $y \in \Lambda$. As in the proof of Theorem 5.4 we can find a transversal $S$ through $y$ and a solution $\sigma$ through $x$ with $\sigma\left(t_{1}\right), \sigma\left(t_{2}\right) \in S$ (for some $t_{1}<t_{2}$ ) and $\sigma\left(t_{1}, t_{2}\right) \cap S=\emptyset$. According to Lemma 4.2 (using the notation from that lemma) and the Jordan Curve Theorem either $\Omega^{+}$or $\Omega^{-}$is a bounded domain enclosed by a Jordan curve. Denote this region by $\Omega$. By Lemma 4.2 , there is a semitrajectory contained in $\mathrm{Cl} \Omega$ and thus there exists a limit set contained in $\mathrm{Cl} \Omega$, therefore bounded. Using Theorem 5.3 we find a stationary point or a periodic trajectory contained in $M$; in the second case we complete the proof applying Lemma 5.11.

REMARK 5.12. The proof of Lenma 5.11 remains correct for a set homeomorphic to the $n$-dimensional closed ball, which generalizes an analogous result for flows (see [2, V.3.7, V.3.8]). Note that the proof given here is simpler than the proof given in [2].

As an immediate consequence of Theorem 5.10 and the compactness of the sphere we get

COROLLARY 5.13. For any semiflow on the 2-dimensional sphere, the sphere contains a stationary point.

\section{REFERENCES}

[1] N. P. Bhatia And O. Hajek, Local Semi-Dynamical Systems, Lecture Notes in Math., vol. 90, Springer-Verlag, 1970.

[2] N. P. Bhatia and G. P. Szegö, Stability Theory of Dynamical Systems, SpringerVerlag, 1370.

[3] K. Ciesielski, Continuity in semidynamical systems, Ann. Polon. Math. 46 (1985), $61-70$.

[4] Kneser type theorems and Baire properties for planar semidynamical systems, Differential Equations: Stability and Control, Lecture Notes in Pure and Appl. Math., vol. 127, Marcel Dekker, 1991, pp. 69-78.

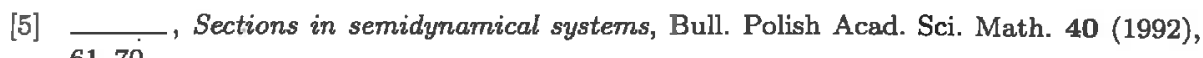
61-70. 
[6] _ Negative Semi-trajectories in Planar Semidynamical Systems, Ph. D. Thesis, Jagiellonian University, Kraków, 1986, (Polish).

[7] K. Ciesielski and K. Omiljanowski, Topological description of cuts in semiflows on 2-manifolds, Topology Appl. (to appear).

[8] S. ELAYDI, Semidynamical systems with nonunique global backward extensions, Funkc. Ekvac. 26 (1983), 173-187.

[9] R. Engelking, General Topology, Polish Scientific Publishers, 1986.

[10] F. HaAs, Poincaré-Bendixson type theorems for two-dimensional manifolds different from the torus, Ann. of Math. 59 (1954), 292-299.

[11] O. HAJEK, Dynamical Systems in the Plane, Academic Press, 1968.

[12] P. Hartman, Ordinary Differential Equations, John Wiley and Sons, 1964.

[13] K. Kuratowski, Topology, vol. II, Academic Press and Polish Scientific Publishers, 1968.

[14] S. Lefschetz, Differential Equations: Geometric Theory, Interscience Publishers, 1957.

[15] R. C. MCCANN, Negative escape time in semidynamical systems, Funkc. Ekvac. 20 (1977), 39-47.

[16] M. H. A. Newman, Elements of the Topology of Plane Sets of Points, Cambridge University Press, 1954.

[17] S. H. SAPErstone, Semidynamical Systems in Infinite Dimensional Spaces, SpringerVerlag, 1981.

[18] A. Schwartz, A generalization of a Poincaré-Bendixson theorem to closed two-dimensional manifolds, Amer. J. Math. 85 (1963), 453-458.

[19] P. Seibert ANd P. Tulley, On dynamical systems in the plane, Arch. Math. (Basel) 18 (1967), 290-292.

[20] E. Serra and M. Tarallo, A new proof of the Poincaté-Bendixson Theotem, Riv. Mat. Pura Appl. 7 (1990), 81-87.

[21] R. SMTrH, Poincaré-Bendixson theory for certain retarded functional differential equations, Differential Integral Equations 5 (1992), 213-240.

[22] R. SRzednickI, On funnel sections of local semiflows, Bull. Polish Acad. Sci. Math. 34 (1986), 203-209.

[23] F. Verhulst, Nonlinear Differential Equations and Dynamical Systems, Springer-Verlag, 1990.

[24] G. T. Whyburn, Amer. Math. Soc. Colloq. Publ., vol. 28 (1963).

Krzysztof Ciesielski

Mathematics Institute

Jagiellonian University

Reymonta 4, 30-059 Kraków, POLAND

E-mail address: ciesiels@im.uj.edu.pl 